\title{
LA PRODUCCIÓN CIENTÍFICA DE ACTA COLOMBIANA DE PSICOLOGÍA: ANÁLISIS DESCRIPTIVO DEL PERIODO 2010-2014
}

\author{
Ernesto L. Ravelo C.*, Susana Mejía, Martha E. González E. \\ Universidad Católica de Colombia
}

Recibido, abril 20/2016

Concepto evaluación, junio 11/2016

Aceptado, junio 12/2016
Referencia: Ravelo Contreras, E. L., Mejía, S. \& González, M. E. (2016). La producción científica de acta colombiana de psicología: análisis descriptivo del periodo 2010-2014. Acta Colombiana de Psicología, 19(2), 281-297. DOI: 10.14718/ACP.2016.19.2.12

\section{Resumen}

\begin{abstract}
Una de las estrategias para la elaboración de los planes de acción o mejoramiento de las publicaciones científicas parte de la mirada retrospectiva o de estudios de caracterización, con respecto a la evolución de estas. Para ello se tienen en cuenta indicadores que exigen las bases e índices de datos, los cuales permiten evaluar la calidad científica, la calidad editorial, la visibilidad, la periodicidad o estabilidad, así como el impacto. En este sentido, el presente artículo tiene como objetivo realizar un análisis descriptivo de los artículos publicados en la Revista Acta Colombiana de Psicología entre los años 2010 y 2014. Se identificaron las variables por tener en cuenta para este análisis: variables categóricas y variables de tipo cuantitativo. El procesamiento y análisis de datos se realizó mediante el paquete IBM SPSS Statistics versión 23; se analizó cada una de las variables categóricas por medio de frecuencias y porcentajes, y las variables cuantitativas aplicando estadísticos descriptivos. Posteriormente se realizaron los cruces entre algunas variables, tanto categóricas como cuantitativas, mediante el uso de frecuencias, porcentajes, medidas descriptivas y algunos gráficos. Se resalta la información relacionada con el número de artículos publicados por año, procedencia de los artículos, tiempos entre la recepción y aceptación de estos porcentajes de idioma de los artículos, tipo de investigaciones de los cuales se derivan los artículos y tipo de análisis estadístico utilizado, entre otros. Palabras clave: producción científica, bibliometría, coautorías, revistas, Psicología
\end{abstract}

\section{SCIENTIFIC PRODUCTION OF COLOMBIAN ACT OF PSYCHOLOGY: DESCRIPTIVE ANALYSIS OF THE PERIOD 2010-2014}

\begin{abstract}
One of the strategies for developing action plans or improving scientific publications originates from a retrospective view or characterization studies, regarding the evolution of such written productions. In order to achieve this, indicators that require databases and indexes are considered. They allow evaluating scientific quality, editorial quality, visibility, periodicity or stability and impact. In this sense, this article aims to make a descriptive analysis of articles published in the "Acta Colombiana de Psicología" (Colombian Act of Psychology) Journal between 2010 and 2014. The variables to be considered for this analysis were identified: categorical variables and quantitative variables. Processing and data analysis was performed using SPSS Statistics IBM package version 23. Each of the categorical variables was analyzed by frequencies and percentages, and quantitative variables using descriptive statistics. Later, crosses between some variables, both categorical and quantitative, were performed using frequencies, percentages, descriptive measures, and some graphics. Information related to the number of articles published per year was highlighted, as well as origin of the articles, time spam between receipt and acceptance, language percentages of the articles, type of research from which articles are derived, and the type of statistical analysis used, among others.

Key words: scientific, bibliometrics, co-authorship, magazines, Psychology.
\end{abstract}

\footnotetext{
* Producto de investigación asociado al proyecto de Bibliometría de las Publicaciones científicas seriadas en la Facultad de Psicología. revistaacta@ucatolica.edu.co
} 


\title{
A PRODUÇÃO CIENTÍFICA DA ACTA COLOMBIANA DE PSICOLOGÍA: ANÁLISE DESCRITIVA DO PERÍODO 2010-2014
}

\begin{abstract}
Resumo
Uma das estratégias para a elaboração dos planos de ação ou melhoramento das publicações científicas parte do olhar retrospectivo ou dos estudos da caracterização a respeito da evolução destas. Para isso, consideram-se os indicadores que exigem as bases e os índices de dados, os quais permitem avaliar a qualidade científica, a qualidade editorial, a visibilidade, a periodicidade ou a estabilidade bem como o impacto. Nesse sentido, o presente artigo tem como objetivo realizar uma análise descritiva dos artigos publicados na revista Acta Colombiana de Psicología entre 2010 e 2014. Identificaram-se as variáveis a considerar para esta análise: variáveis categóricas e variáveis de tipo quantitativo. O processamento e a análise de dados foi realizada mediante o IBM SPSS Statistics versão 23; foi analisada cada uma das variáveis categóricas por meio de frequências e porcentagens, e as variáveis quantitativas aplicando estatísticos descritivos. Em seguida, foram realizados os cruzamentos entre algumas variáveis, tanto categóricas quanto quantitativas, mediante o uso de frequências, porcentagens, medidas descritivas e alguns gráficos. Ressalta-se a informação relacionada com o número de artigos publicados por ano, a procedência dos artigos, o tempo entre a recepção e a aceitação deles, as porcentagens de idioma dos artigos, os tipos de pesquisa das quais se derivam os artigos e o tipo de análise estatística utilizada, entre outros.

Palavras-chave: produção científica, bibliometria, coautoria, revistas, Psicologia.
\end{abstract}

Las publicaciones científicas seriadas se han consolidado como el medio por excelencia que permite la difusión de la ciencia para la utilización del conocimiento (Vessuri, 1995; Patalano, 2005). La visibilidad institucional y la divulgación del conocimiento a partirde las publicaciones científicas son cada vez de mayor importancia en el mundo de la ciencia y de la academia (Ravelo, 2007). Según Sánchez López y Vidal Vásquez (2014), "las revistas científicas representan un enlace en el proceso de comunicación y divulgación de la producción científica de cada ámbito académico, científico y profesional, las cuales validan y certifican el conocimiento y comunican a toda la sociedad científica un nuevo avance y/o aportación en su campo específico para su conocimiento, rechazo o aceptación" (p. 2).

Así mismo, realizar estudios descriptivos, bibliométricos y análisis de las publicaciones científicas seriadas es de suma importancia para su caracterización. Por ejemplo, en psicología se han realizado estudios por parte de Fava y Montonary (1997); Fava, Ottolini y Sonino (2001); Rahman y Fukui (2000); Rivera-Garzón (2008). En Colombia han llevado a cabo análisis de este tipo los siguientes autores: Ávila-Toscano, Marenco-Escudero y Madariaga (2014); Puche-Navarro y Ossa (2012); López-López y Calvache (1998); Jaraba-Barrios, Guerrero-Castro, Gómez-Morales y López-López (2011). Si se tiene en cuenta su evolución, en América Latina se ha mejorado la producción de las revistas en cuanto a su alta calidad editorial y científica (Buela-Casal y López-López, 2005; Zych y Buela-Casal, 2007; 2010; López-López, García-Cepero, Aguilar-Bustamante, Silva y Aguado-López, 2010).

Pero no solamente el cumplimiento de las características editoriales y la calidad científica permiten cualificar a una publicación científica, sino que además es necesario la inclusión en índices y bases de datos para ganar visibilidad, prestigio y posicionamiento nacional e internacional en las comunidades científicas. Con la indexación se promueve el incremento de artículos publicados que reúnen las características, indicadores y tendencias de las publicaciones científicas, y la participación internacional de autores en los artículos (Buela-Casal, Zych, Sierra y Bermúdez, 2007; García Martínez, Guerrero Bote, Vargas Quesada, y De Moya-Anegón, 2008; Zych y Buela-Casal, 2007, 2010), entre otros indicadores.

En 1998 la Facultad de Psicología de la Universidad Católica de Colombia comenzó a editar la Revista Acta Colombiana de Psicología con la finalidad de contribuir a la visibilidad y divulgación de los resultados de la investigación institucional, nacional e internacional. En cumplimiento de su misión, Acta publica hallazgos originales de las investigaciones en Psicología, y de esta disciplina en diálogo con otras, con el propósito de divulgarlas a la comunidad universitaria y a todas las personas interesadas en conocer sus nuevos avances y aplicaciones a distintos campos y necesidades de la sociedad contemporánea.

En este sentido, el interés de Acta Colombiana de Psicología ha sido la publicación de artículos de alta calidad científica de acuerdo con las exigencias nacionales e internacionales en cuanto al cumplimiento de características editoriales, logrando cada vez mayor visibilidad y posicionamiento nacional e internacional. En cuanto a lo nacional, se destaca su indexación en la categoría A1 del Índice Bibliográfico Nacional-Publindex (año 2008), reconocimiento obtenido por su alta calidad científica y su inclusión en diferentes sistemas de indexación reconocidos mundialmente; hasta la 
fecha Acta se encuentra incluida e indexada en PsycINFO, Scopus, SciELO, Redalyc, Lilacs, Ulrich's, Iresie, EBSCO, Imbiomed, DOAJ, Latindex, Clase, Psicodoc, entre otras bases e índices de datos internacionales.

Por otra parte, se han realizado convenios colaborativos de cooperación entre Acta Colombiana de Psicología y las revistas internacionales, Revista Mexicana de Análisis de la Conducta y Revista Latinoamericana de Medicina Conductual y Journal of Behavior, Health y Social Issues. Estos han permitido que las revistas construyan, fortalezcan y consoliden redes académicas entre autores y árbitros, como también aumenten su cobertura en divulgación para propiciar mayor visibilidad e incremento en las estadísticas de citación, entre otros muchos beneficios que se obtienen al trabajar en red, tal como lo sostienen algunos estudios (Russell, Ainsworth y Narváez-Berthelemot, 2006).

De igual manera, se han editado en colaboración los siguientes números con énfasis especial: Volumen (Vol.) 16, número (No) 2 (2013), con el Centro Regional de Investigaciones Multidisciplinarias, Programa de Investigación Equidad y Género, Cuernavaca, Morelos, México. Universidad Nacional Autónoma de México, Facultad de Estudios Superiores Iztacala, Proyecto Aprendizaje Humano, Estado de México, México; el Vol. 17 No. 2 (2014), con la Red de Psicobiología y Neuropsicología del Sistema Mexicano de Investigación en Psicología (SMIP); el Vol. 18 No. 1 (2015), con la Red Nacional de Psicología Experimental Humana (RMIPEH) del Sistema Mexicano de Investigación en Psicología (SMIP); el Vol. 18 No. 2 (2015), con la Red Mexicana de Investigación en Psicología Educativa del Sistema Mexicano de Investigación en Psicología (SMIP) y para el Vol. 19 No. 1 (2016) con el Nodo de Psicología y Salud y el Nodo de Psicología Clínica de la Red de Investigadores de Ascofapsi.

Con la finalidad de caracterizar lo publicado en Acta, el presente artículo tiene como objetivo presentar un panorama general de la producción publicada en la revista Acta Colombiana de Psicología durante un periodo de cinco años, en respuesta a la necesidad de identificar indicadores descriptivos a partir de los artículos publicados entre 2010 y 2014.

\section{MÉTODO}

\section{Universo de estudio}

Se revisó la totalidad de los artículos publicados en Acta Colombiana de Psicología desde el año 2010, a partir del Vol. 13 No.1, hasta lo editado en 2014 Vol. 17 Nos.1 y 2. Durante este periodo de cinco años se publicaron 125 artículos que fueron analizados teniendo en cuenta variables categóricas y variables de tipo cuantitativo, para lo cual se calculó la media y la desviación estándar.

\section{Instrumentos}

Para la recolección de la información se construyó una base de datos a partir de una plantilla creada en el programa Excel. La base fue alimentada teniendo en cuenta todos los artículos publicados en la Revista Acta Colombiana de Psicología en el periodo 2010 a 2014.

\section{Procedimiento}

Inicialmente se identificaron las variables por tener en cuenta en el análisis descriptivo-bibliométrico. Se elaboró una plantilla en el programa Excel y posteriormente, a partir de la consulta de cada uno de los números publicados semestralmente, se identificaron y levantaron los datos de las variables con la finalidad de construir la base de datos para llevar a cabo el procesamiento y análisis de la información.

\section{Análisis de datos}

Una vez completada la base de datos, se llevó a cabo el procesamiento y análisis de datos mediante el paquete IBM SPSS Statistics versión 23. En primer lugar, se analizó cada una de las variables categóricas por medio de frecuencias y porcentajes y las variables cuantitativas aplicando estadísticos descriptivos. Seguidamente se realizaron los cruces por áreas de conocimiento, mediante el uso de frecuencias, porcentajes, medidas descriptivas y algunos gráficos.

\section{RESULTADOS Y DISCUSIÓN}

A continuación se describen los resultados del análisis de los artículos científicos de la revista Acta Colombiana de Psicología, durante el periodo comprendido entre 2010 y 2014. En primer lugar, se muestran los análisis para cada una de las variables categóricas del estudio por medio de frecuencias y/o porcentajes y para las variables de tipo cuantitativo la media y la desviación estándar. En segundo lugar, se presentan los cruces de variables por áreas de conocimiento, por medio de tablas de contingencia y promedio para la variable número de autores.

\section{Resultados descriptivos por variable}

Análisis variables categóricas o cualitativas

Como se muestra en la Tabla 1, entre 2010 y 2014 la Revista Acta Colombiana de Psicología ha publicado 125 artículos, el número de artículos por año varía entre 23 (2012) y 27 (2014). 
Tabla 1.

Publicaciones por año

\begin{tabular}{cc}
\hline Año & Número de artículos \\
\hline 2010 & 26 \\
2011 & 24 \\
2012 & 23 \\
2013 & 25 \\
2014 & 27 \\
\hline Total & 125 \\
\hline
\end{tabular}

La Figura 1 muestra que el $60.5 \%$ de los autores que han publicado durante los cinco años de observación corresponden a investigadores internacionales; el 25.8\% a autores nacionales externos a la Universidad Católica de Colombia, mientras que el $4.8 \%$ son investigaciones realizadas por docentes de la institución.

En cuanto al idioma, el $80 \%$ de los artículos se publicó en idioma español; el $11.2 \%$, en inglés, y el $8.8 \%$, en portugués (véase Figura 2).

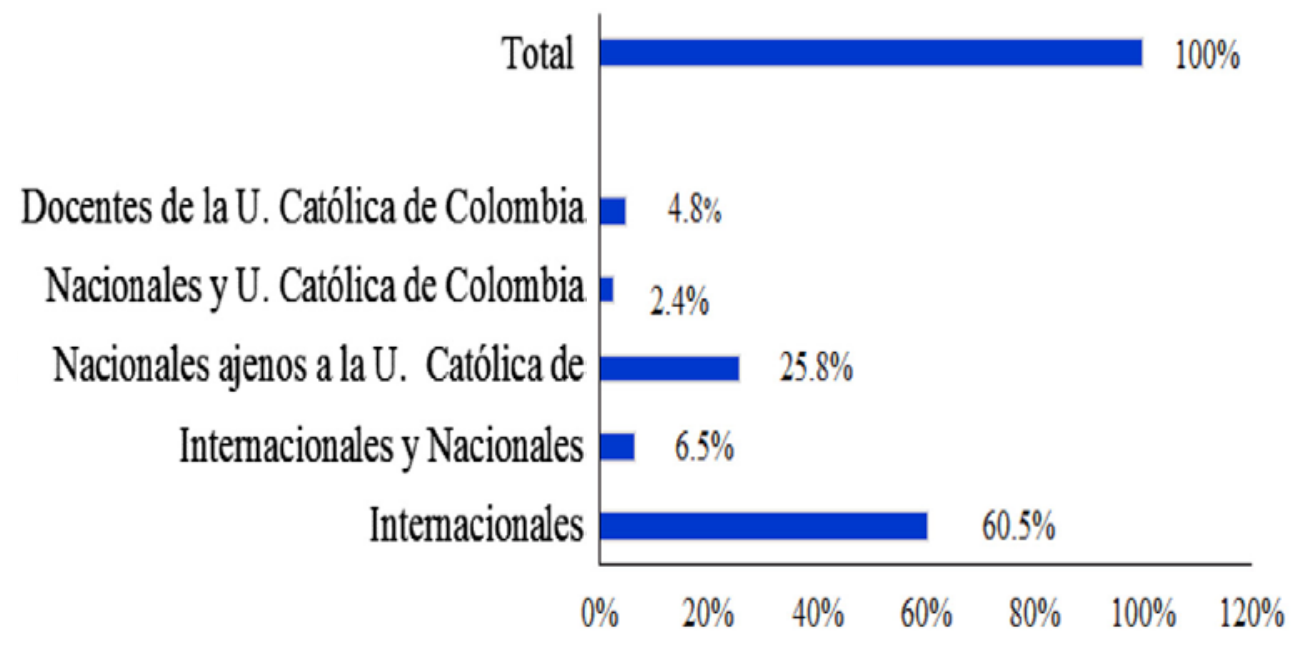

$\underline{\text { Figura 1. Procedencia institucional de los autores }}$

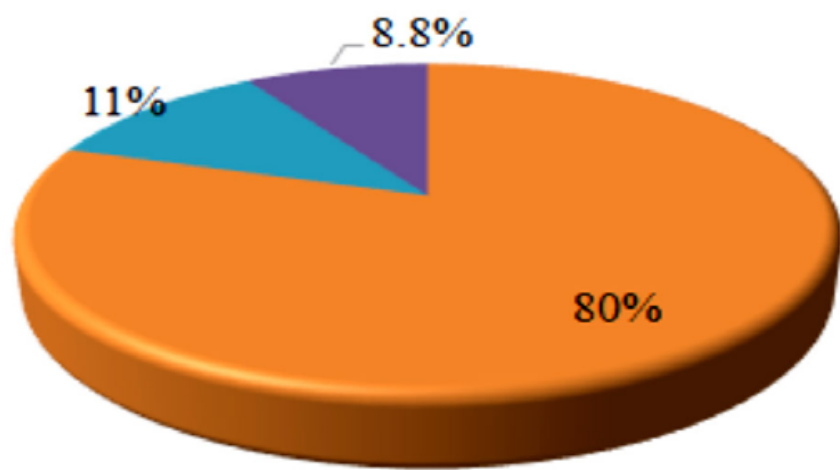

日español

a Inglés

国 Portugués

Figura 2. Porcentaje de idioma de publicación 
De acuerdo con el área de conocimiento, el 38.4\%, corresponde a artículos publicados en el área de la psicología clínica y de la salud, seguida por el área social-comunitaria con un $20.8 \%$. En tercer lugar, con un $15.2 \%$ se encuentra el área educativa, mientras que el área de psicometría está representada con el $12.8 \%$. Con baja representación se encuentra el área de neuropsicología, con el 4\%, adicciones y organizacional con el $3.2 \%$, jurídica con $0.8 \%$, que corresponde a un artículo y, finalmente, con el $1.6 \%$, los estudios bibliométricos (véase Figura 3).
Con respecto al tipo de investigaciones publicadas, el $87.3 \%$ corresponde a estudios cuantitativos, el $10 \%$ a estudios cualitativos y un $2.7 \%$ a metodologías mixtas (véase Figura 4).

En cuanto al tipo de muestreo, los autores no lo registran en el $43.2 \%$ de los artículos, el $32.8 \%$ aplicó un muestreo probabilístico, y el $24 \%$ un muestreo no probabilístico (véase Figura 5).

En relación con los instrumentos utilizados, los más usados fueron las pruebas psicológicas, las entrevistas y las encuestas con un $70.6 \%, 20.3 \%$ y $11 \%$, respectivamente (véase Figura 6).

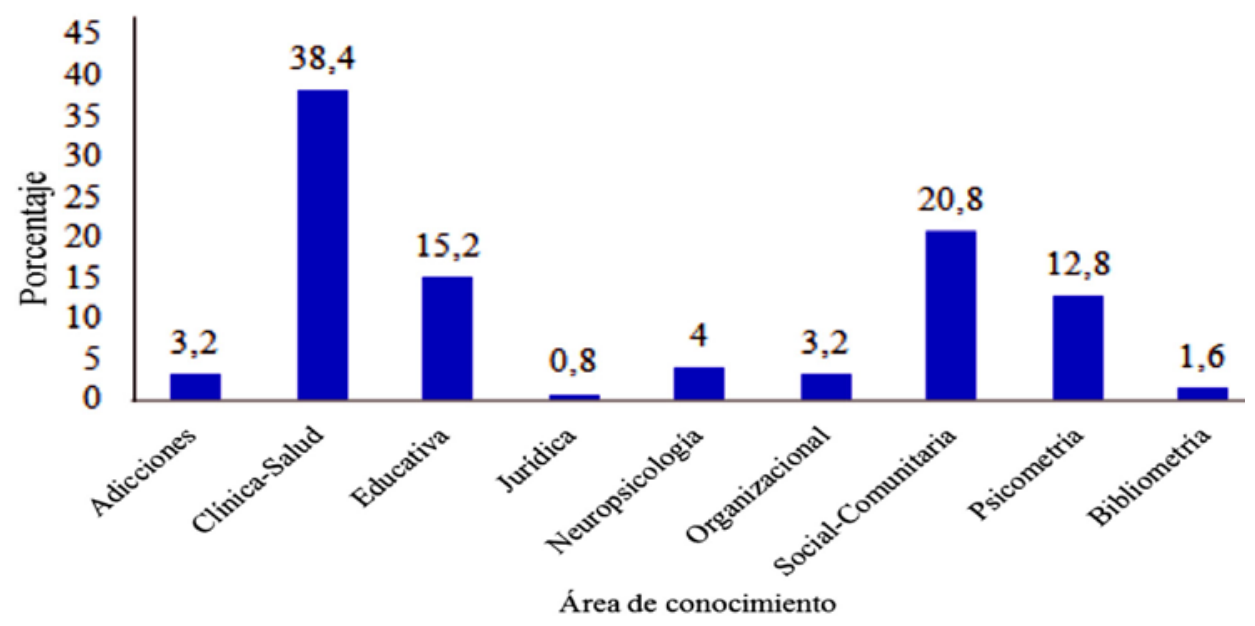

Figura 3. Publicaciones por área de conocimiento

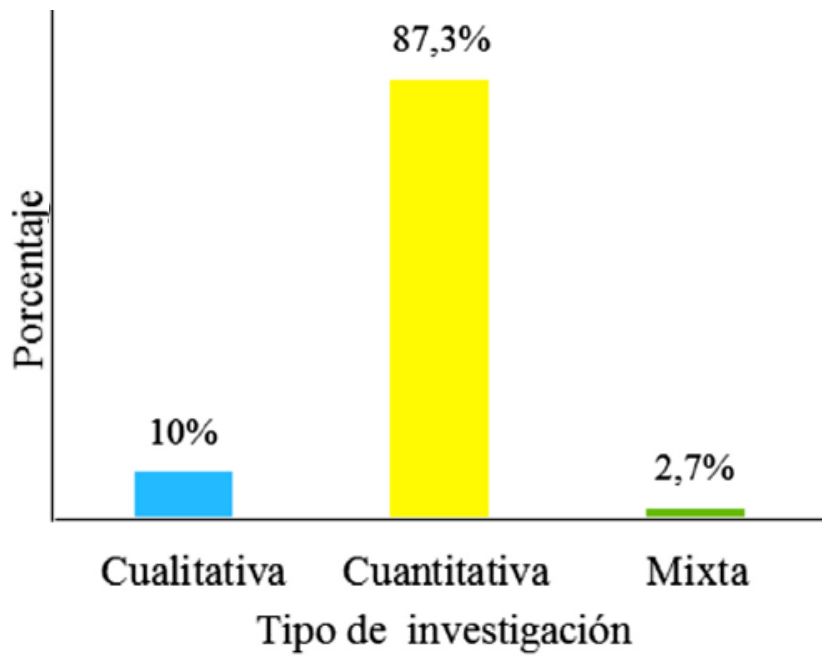

Figura 4. Publicaciones según tipo de investigación

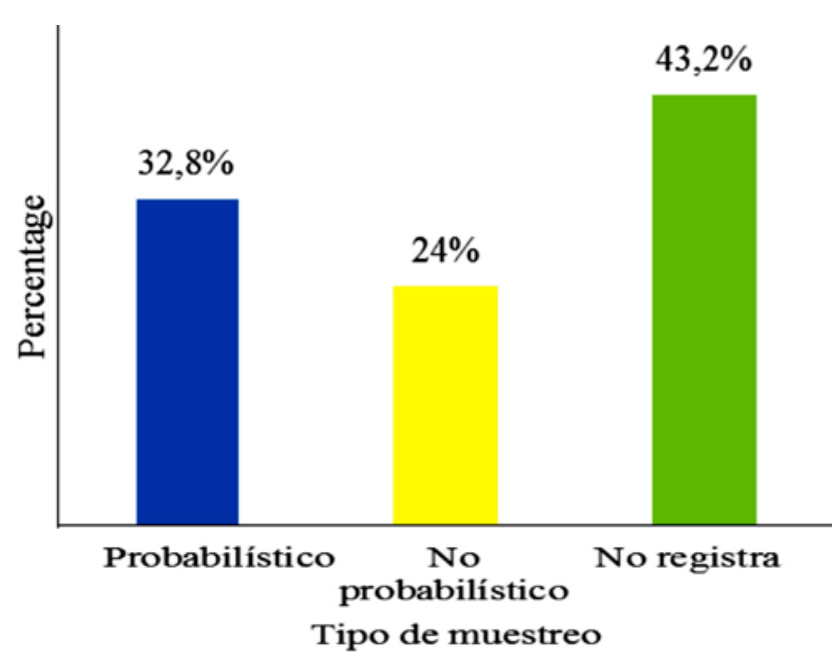

Figura 5. Publicaciones según tipo de muestreo 


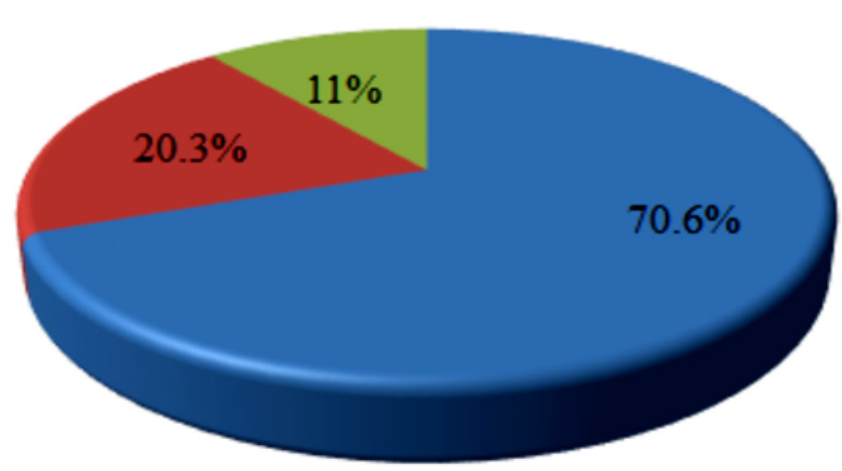

\section{a Pruebas}

Entrevistas

日Encuestas

Figura 6. Publicaciones según uso de instrumentos

Por otro lado, se encontró que 101 de las 12 investigaciones llevaron a cabo análisis estadísticos, siendo los más frecuentes los análisis descriptivos con un $82.2 \%$, seguido por los análisis inferenciales con un $72.3 \%$. Así mismo, se encontró que el $66.3 \%$ de las investigaciones realizaron pruebas paramétricas, mientras que las pruebas no paramétricas, por su parte, fueron aplicadas en el $22.8 \%$ de los casos. Los resultados se muestran en la Figura 7.
Análisis de variables cuantitativas

Se examinaron 125 artículos; los resultados del análisis de las variables cuantitativas se presentan a continuación.

En promedio, el número de autores por artículo es de dos o tres; en cuanto a la diferencia entre el tiempo de recibido el artículo y el concepto, se encuentra un promedio de 190 $( \pm 122)$ días, mientras que el promedio entre el concepto y la aceptación es de 61 ( \pm 66 ) días (véase Tabla 2).



Figura 7. Publicaciones según tipo de análisis estadístico

Tabla 2.

Estadísticos descriptivos

\begin{tabular}{lcccc}
\hline & Mínimo & Máximo & Media & Desv. típ. \\
\hline Número de autores & 1 & 10 & 2.64 & 1.478 \\
Tiempo recibido-concepto (días) & 8 & 563 & 189.50 & 121.941 \\
Tiempo recibido-aceptado (días) & 9 & 696 & 250.66 & 146.224 \\
Tiempo concepto-aceptación (días) & 0 & 305 & 61.16 & 66.376 \\
Número de referencias & 7 & 107 & 39.22 & 15.704 \\
\hline
\end{tabular}




\section{Cruces de variables}

\section{Cruces por años}

Una vez realizados los análisis descriptivos para cada una de las variables se llevó a cabo la comparación por años.

De acuerdo con los datos registrados en la Figura 8, durante los cinco años de observación (2010-2014), ha predominado la publicación de artículos en idioma español, aunque llama la atención que en 2013, el 40\% fueron escritos en inglés.

Así mismo, se evidencia que predominan las publicaciones de autores internacionales con un total de $60.5 \%$ (75 artículos), seguido por autores colombianos ajenos a la Universidad Católica de Colombia con el 25.8\% (véase Figura 9).

En la Figura 10 se describen las publicaciones por año de acuerdo con el área de conocimiento, siendo las especialidades de psicología clínica y de la salud las más frecuentes, con 48 artículos publicados.

En cuanto al tipo de muestreo, predomina el uso del muestreo probabilístico (véase Figura 11). En cuanto al tipo de investigación, la que más se presenta es la investigación cuantitativa con un total de 96 artículos (véase Figura 12) y descriptiva (véase Figura 13).

Por otro lado, entre el $25.9 \%$ y el 55\% de las investigaciones empleó análisis correlaciónales (véase Figura 14).

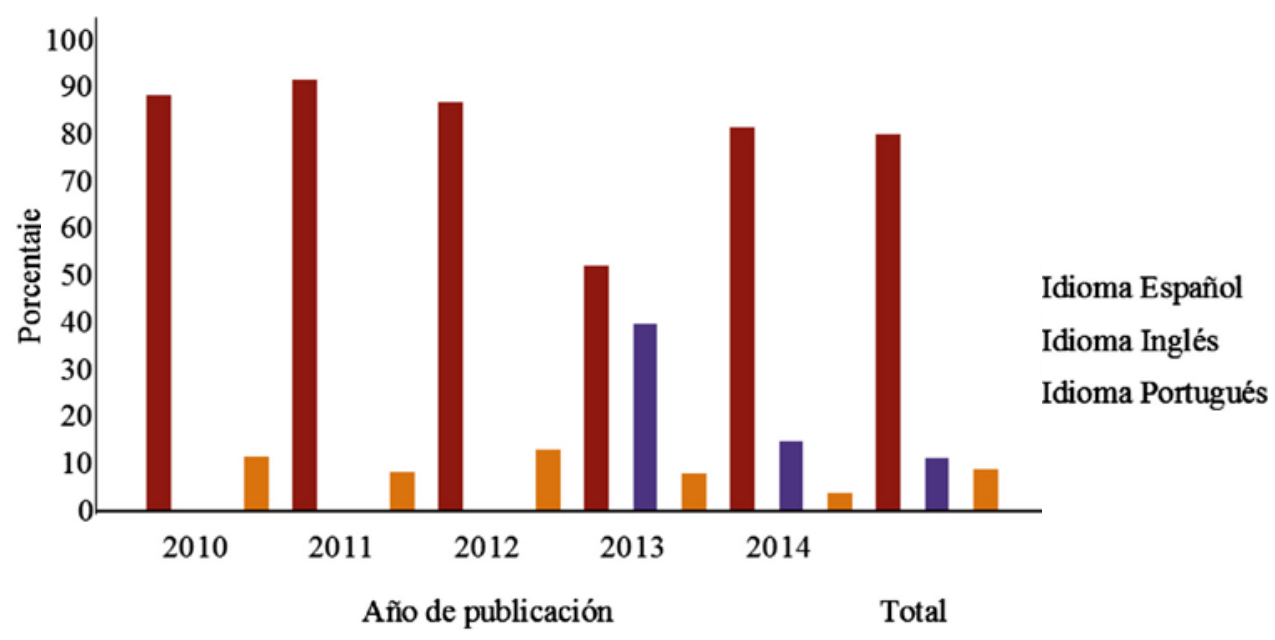

Figura 8. Idioma de publicación por año

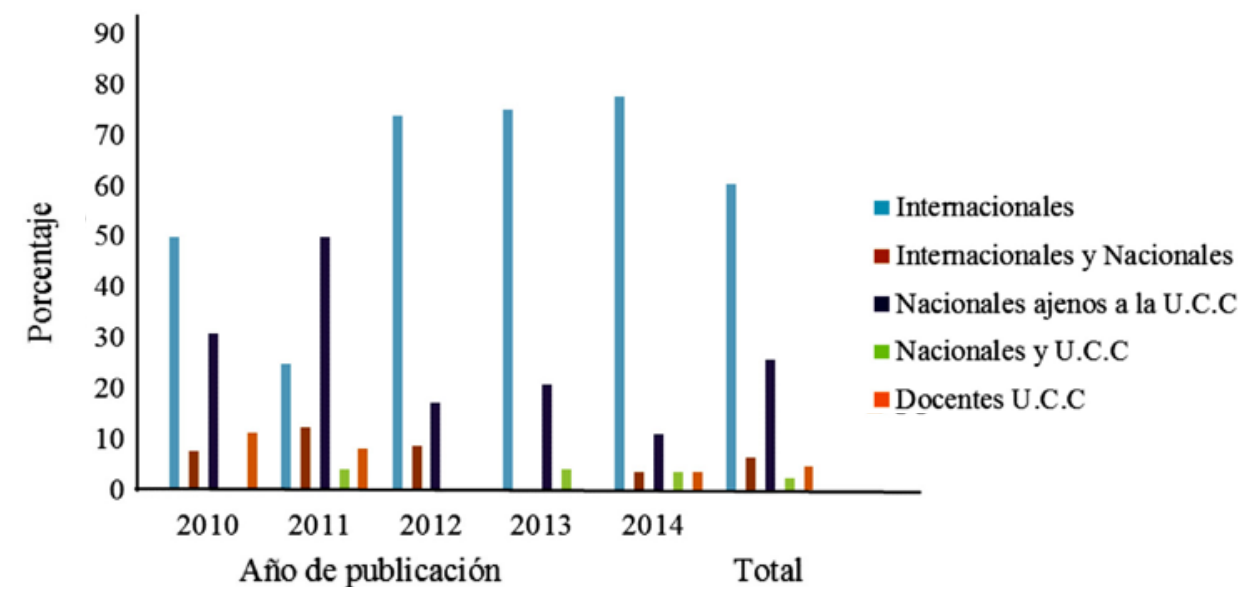

Figura 9. Procedencia de autores por año 


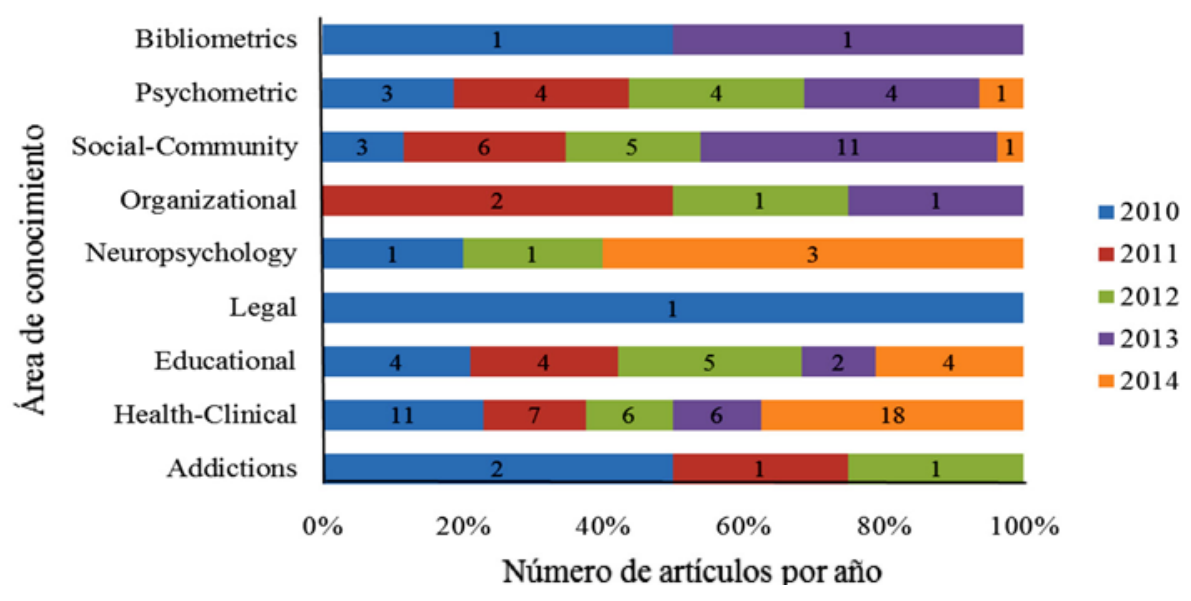

Figura 10. Publicaciones por año de acuerdo con el área de conocimiento

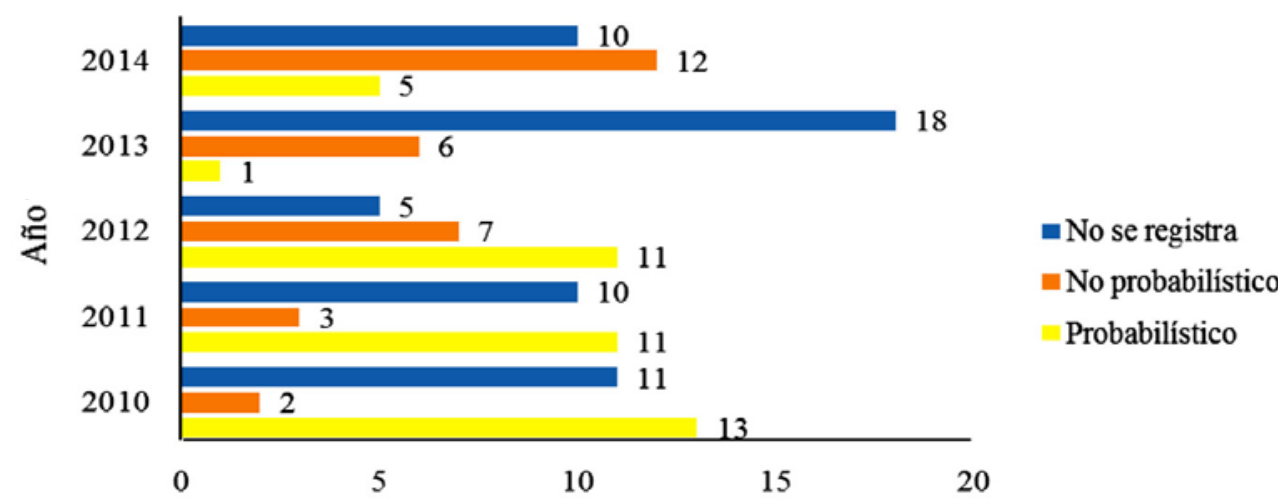

Número de artículos

Figura 11. Publicaciones anuales por tipo de muestreo

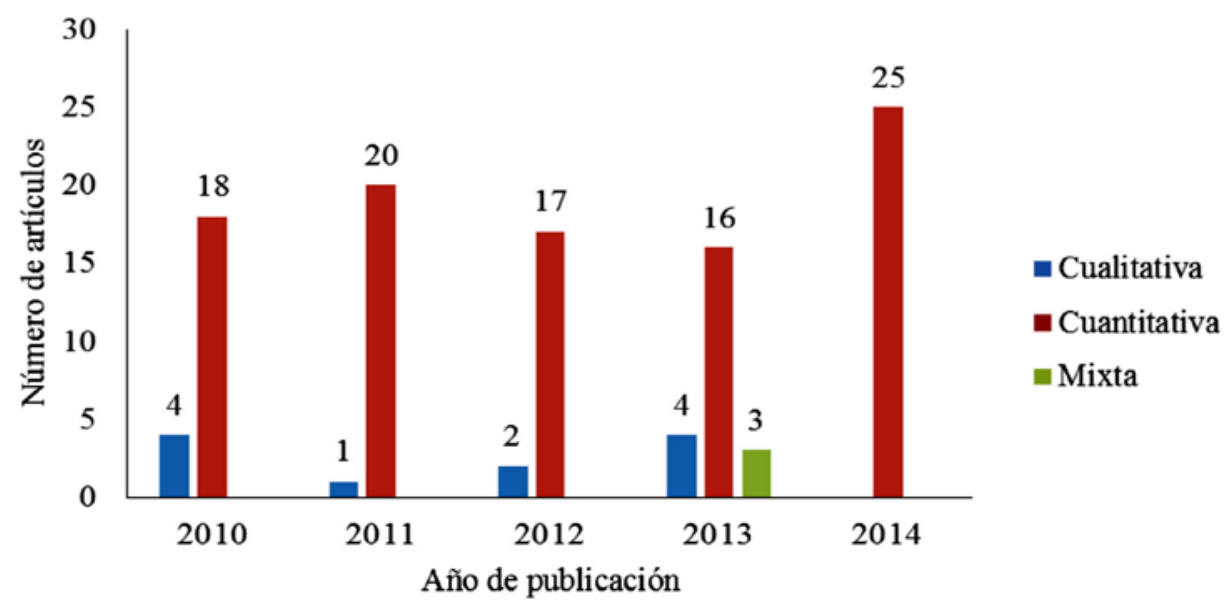

Figura 12. Publicaciones anuales por tipo de investigación 


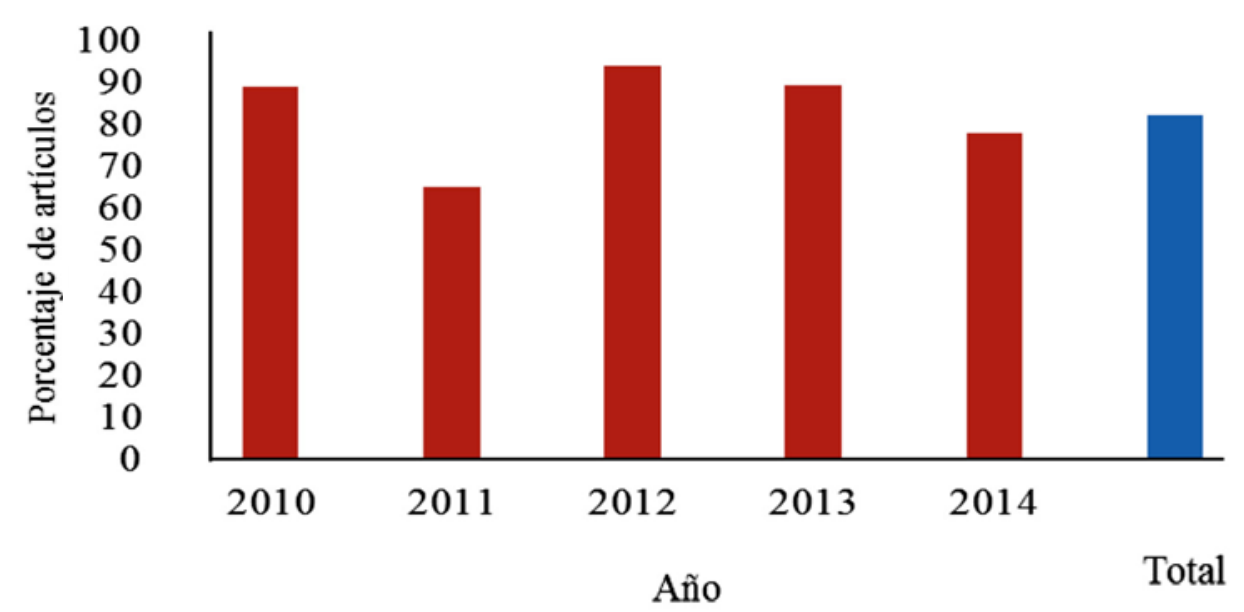

Figura 13. Uso de análisis descriptivos por año

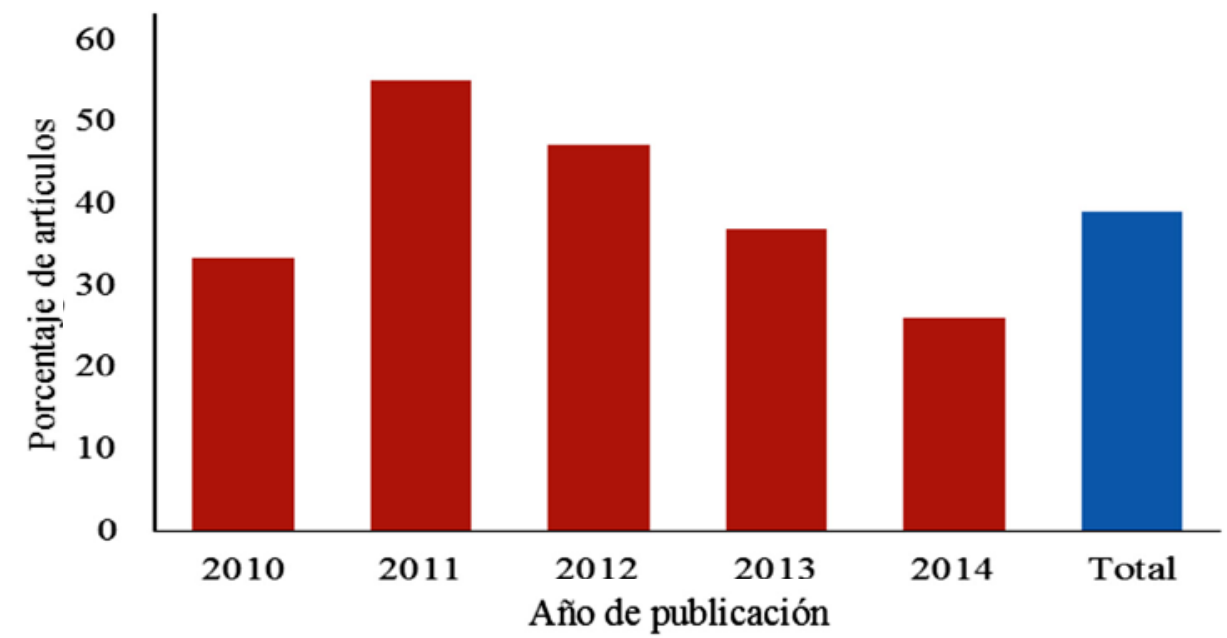

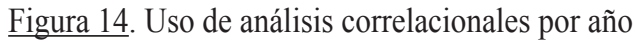

Los análisis inferenciales fueron los usados para dar respuesta al objetivo de investigación desde el 50\% (año 2011) hasta el 94.1\% (2012) (véase Figura 15).

El mayor porcentaje de artículos que usó métodos multivariados como análisis estadístico se presentó en el 2012, con el 58.8\%, seguido por el 2013 con el 47.4\%, como se presenta en la Figura 16.
Las pruebas paramétricas son utilizadas en el 50\% o más de los artículos publicados en los últimos cinco años, mientras que los análisis no paramétricos se usaron en el $30 \%$ de los artículos del año 2011, seguidos por el $27.8 \%$ del año 2010 (véase Tabla 3). 


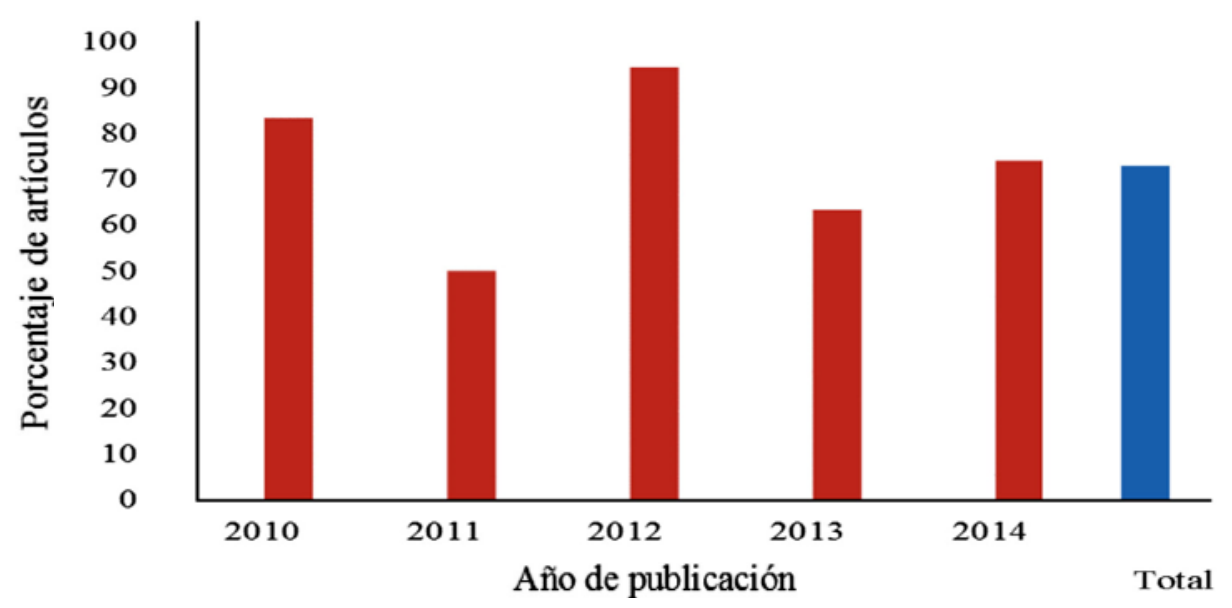

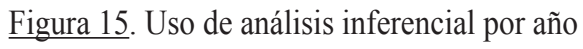

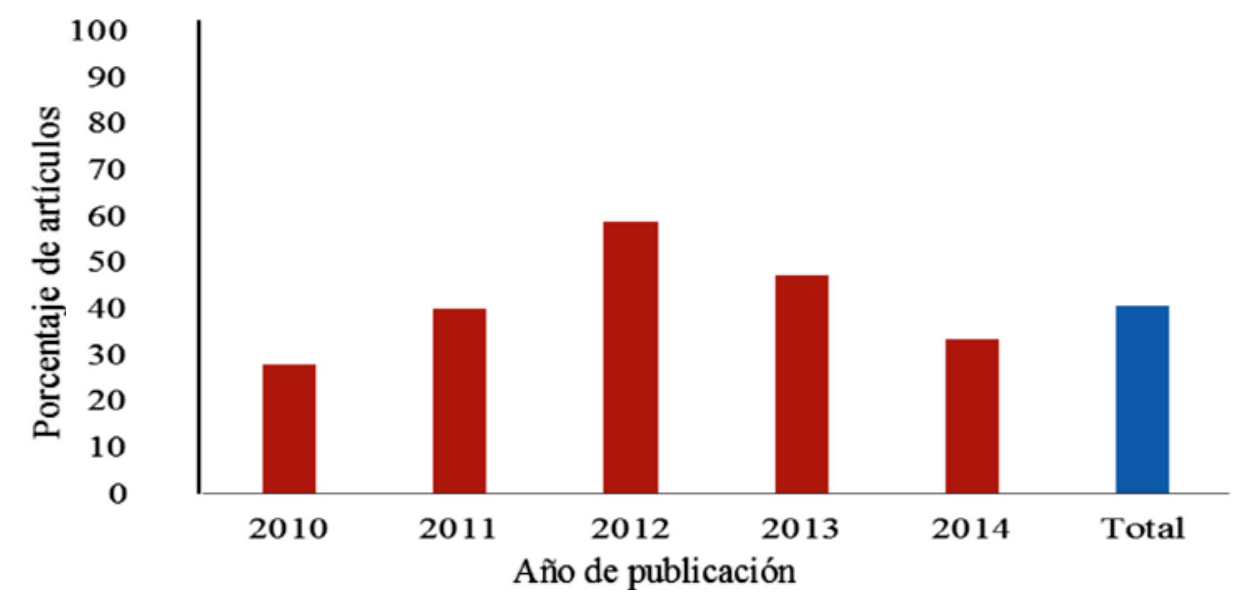

Figura 16. Frecuencia del uso de análisis multivariado por año

Tabla 3.

Uso de pruebas paramétricas y no paramétricas por año

\begin{tabular}{|c|c|c|c|c|c|c|c|}
\hline & & & & Año & & & Total \\
\hline & & 2010 & 2011 & 2012 & 2013 & 2014 & \\
\hline \multirow[t]{4}{*}{ Uso de pruebas paramétricas } & No & 9 & 5 & 2 & 8 & 10 & 34 \\
\hline & & $(50.0 \%)$ & $(25.0 \%)$ & $(11.8 \%)$ & $(42.1 \%)$ & $(37.0 \%)$ & $(33.7 \%)$ \\
\hline & Sí & 9 & 15 & 15 & 11 & 17 & 67 \\
\hline & & $(50.0 \%)$ & $(75.0 \%)$ & $(88.2 \%)$ & $(57.9 \%)$ & $(63.0 \%)$ & $(66.3 \%)$ \\
\hline \multirow[t]{4}{*}{ Uso de pruebas no paramétricas } & No & 13 & 14 & 14 & 15 & 22 & 78 \\
\hline & & $(72.2 \%)$ & $(70.0 \%)$ & $(82.4 \%)$ & $(78.9 \%)$ & $(81.5 \%)$ & $(77.2 \%)$ \\
\hline & Sí & 5 & 6 & 3 & 4 & 5 & 23 \\
\hline & & $(27.8 \%)$ & $(30.0 \%)$ & $(17.6 \%)$ & $(21.1 \%)$ & $(18.5 \%)$ & $(22.8 \%)$ \\
\hline
\end{tabular}


Cruces por área de conocimiento

Además de las comparaciones por año, se realizó el análisis por área de conocimiento, cuyos resultados se presentan a continuación:

En la Figura 17 se evidencia que prevalece la publicación en español, aunque se resalta la publicación en inglés en al área de neuropsicología, con un 40\%, y en el área social-comunitaria, con el $26.9 \%$.

Por otro lado, sobresale que en la mayoría de las áreas los artículos son escritos por autores internacionales, con porcentajes que oscilan entre el $46.7 \%$ y el $80 \%$. Así mismo, en psicometría el $46.7 \%$ de artículos son de autores nacionales externos a la Universidad Católica de Colombia (véase Tabla 4).

En todas las áreas predomina el uso de investigación cuantitativa, sin embargo, sobresale en el área Social-Comunitaria la investigación cualitativa en el $42 \%$ de los artículos.

En cuanto al tipo de muestreo predomina el probabilístico en las siguientes áreas: adicciones, psicología educativa, jurídica y psicometría; el no probabilístico en el área clínica y salud (véase Figura 18). Así mismo, son más frecuentes las investigaciones de tipo cuantitativo, aunque el $42.1 \%$ de los artículos del área social-comunitario son cualitativos (véase Figura 19).

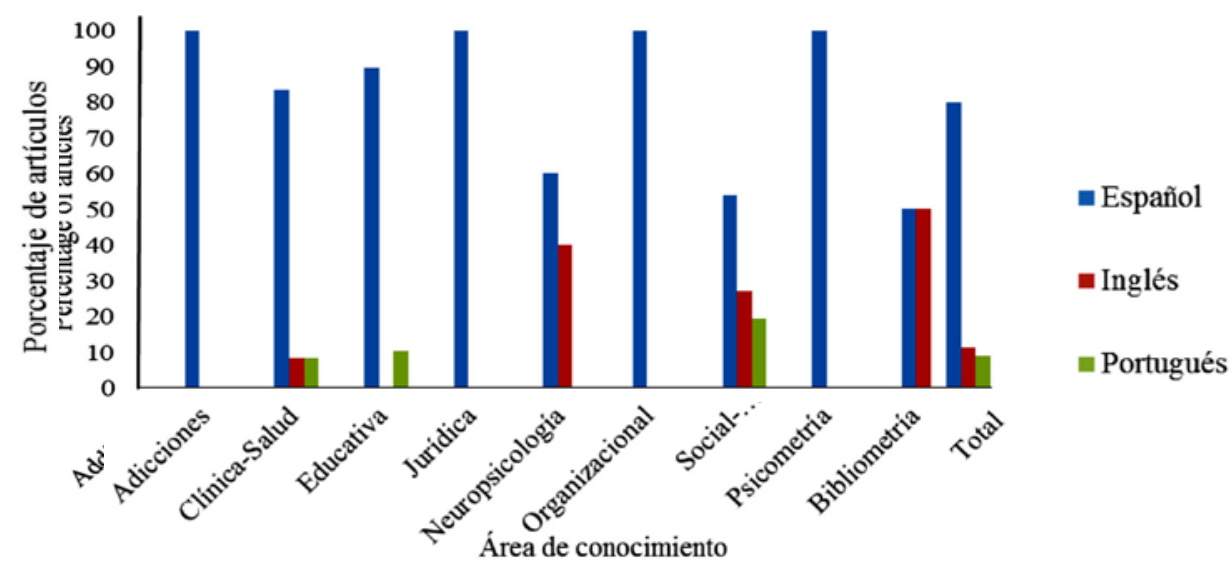

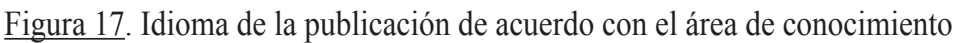

Tabla 4.

Procedencia de los autores de acuerdo con el área de conocimiento

\begin{tabular}{|c|c|c|c|c|c|c|}
\hline & & & & Tipo de autores & & \\
\hline & & Internacionales & $\begin{array}{l}\text { Internacionales } \\
\text { y Nacionales }\end{array}$ & $\begin{array}{c}\text { Nacionales externos } \\
\text { a la UCC }\end{array}$ & Nacionales UCC & Docentes UCC \\
\hline & Adicciones & $\begin{array}{c}2 \\
(50.0 \%)\end{array}$ & 0 & $\begin{array}{c}1 \\
(25.0 \%)\end{array}$ & 0 & $\begin{array}{c}1 \\
(25.0 \%)\end{array}$ \\
\hline & Clínica- Salud & $\begin{array}{c}31 \\
(64.6 \%)\end{array}$ & $\begin{array}{c}3 \\
(6.2 \%)\end{array}$ & $\begin{array}{c}10 \\
(20.8 \%)\end{array}$ & $\begin{array}{c}2 \\
(4.2 \%)\end{array}$ & $\begin{array}{c}2 \\
(4.2 \%)\end{array}$ \\
\hline & Educativa & $\begin{array}{c}11 \\
(57.9 \%)\end{array}$ & $\begin{array}{c}1 \\
(5.3 \%)\end{array}$ & $\begin{array}{c}7 \\
(36.8 \%)\end{array}$ & 0 & 0 \\
\hline & Jurídica & 0 & 0 & $\begin{array}{c}1 \\
(100.0 \%)\end{array}$ & 0 & 0 \\
\hline $\begin{array}{l}\text { Área de } \\
\text { conocimiento }\end{array}$ & Neuropsicología & $\begin{array}{c}4 \\
(80.0 \%)\end{array}$ & 0 & $\begin{array}{c}1 \\
(20.0 \%)\end{array}$ & 0 & 0 \\
\hline & Organizacional & 0 & $\begin{array}{c}1 \\
(25.0 \%)\end{array}$ & $\begin{array}{c}2 \\
(50.0 \%)\end{array}$ & 0 & $\begin{array}{c}1 \\
(25.0 \%)\end{array}$ \\
\hline & Social-Comunitaria & $\begin{array}{c}19 \\
(73.1 \%)\end{array}$ & $\begin{array}{c}1 \\
(3.8 \%)\end{array}$ & $\begin{array}{c}3 \\
(11.5 \%)\end{array}$ & $\begin{array}{c}1 \\
(3.8 \%)\end{array}$ & $\begin{array}{c}2 \\
(7.7 \%)\end{array}$ \\
\hline & Psicometría & $\begin{array}{c}7 \\
(46.7 \% 9)\end{array}$ & $\begin{array}{c}1 \\
(6.7 \%)\end{array}$ & $\begin{array}{c}7 \\
(46.7 \%)\end{array}$ & 0 & 0 \\
\hline & Bibliometría & $\begin{array}{c}1 \\
(50.0 \%) \\
\end{array}$ & $\begin{array}{c}1 \\
(50.0 \%) \\
\end{array}$ & 0 & 0 & 0 \\
\hline Total artículos & & 75 & 8 & 32 & 3 & 6 \\
\hline
\end{tabular}




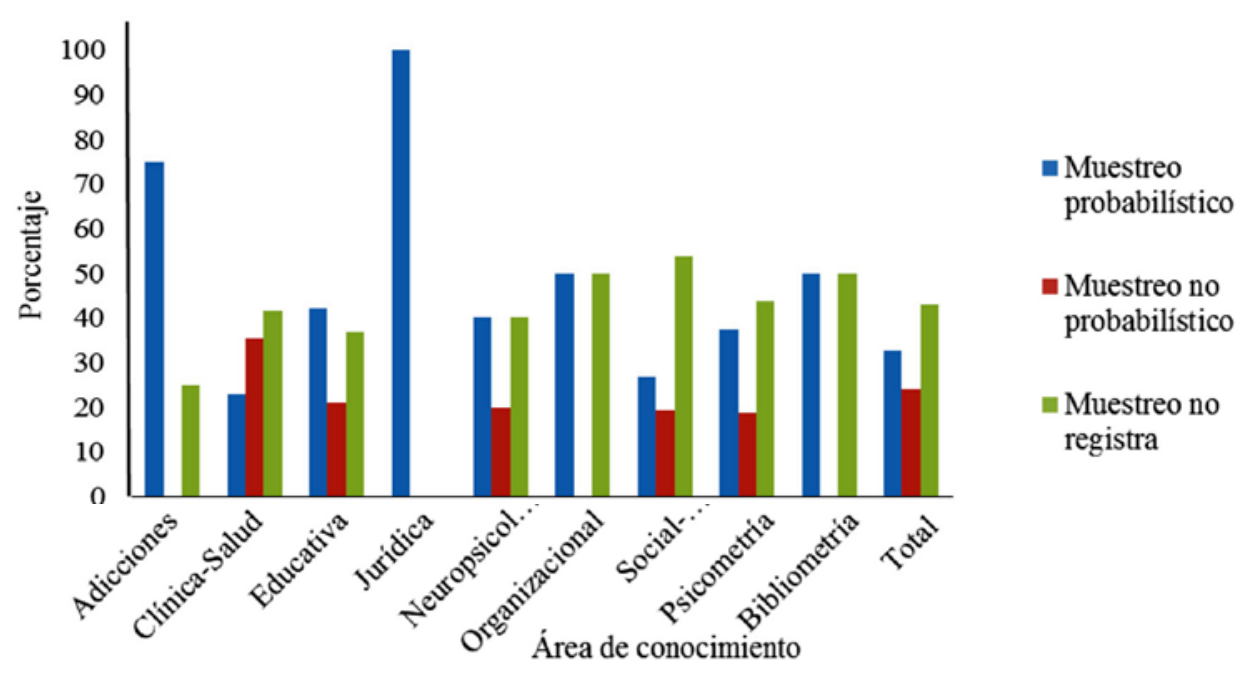

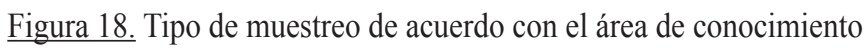

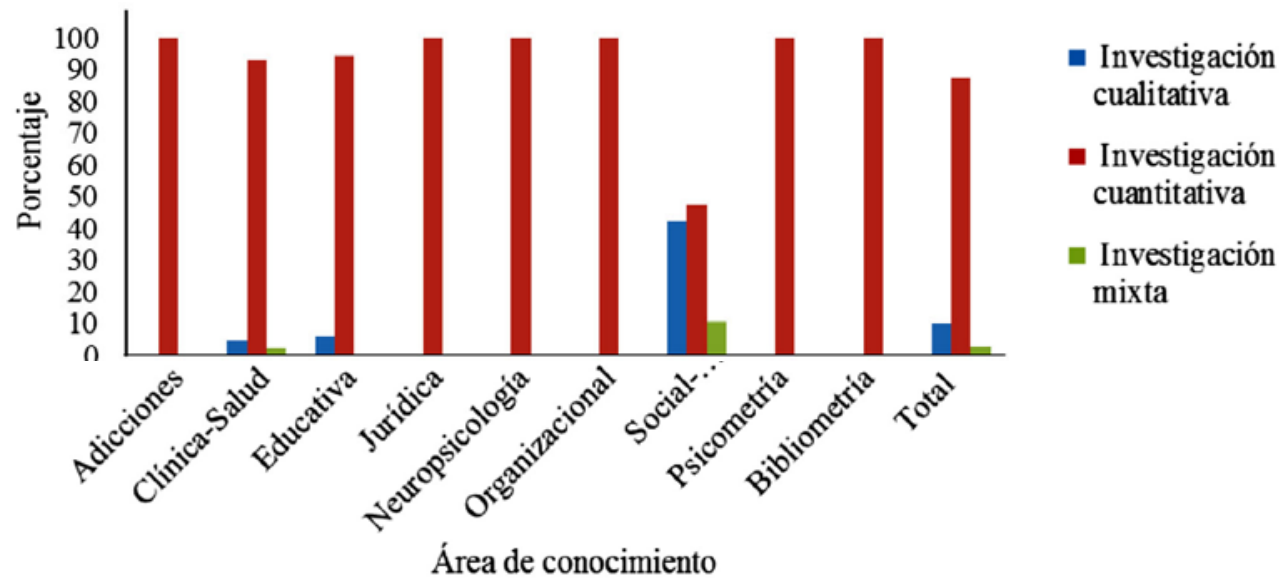

Figura 19. Tipo de investigación de acuerdo con el área de conocimiento

En cuanto a los análisis, los descriptivos son aplicados en todas las áreas, con porcentajes entre 60\% y 100\% (véase Tabla 5).

Los análisis correlacionales, por su parte, son usados en todas las áreas excepto en bibliometría y jurídica (véase Tabla 6).
La aplicación de análisis estadísticos inferenciales se presenta en todas las áreas excepto en bibliometría (véase Tabla 7). 
Tabla 5.

Uso de análisis descriptivos de acuerdo con el área de conocimiento

\begin{tabular}{llcc}
\hline & & \multicolumn{2}{c}{ Uso de análisis descriptivos } \\
\cline { 3 - 4 } & & No & Sí \\
& Adicciones & 1 & 3 \\
& Clínica-Salud & $(25.0 \%)$ & $(75.0 \%)$ \\
& & 5 & 37 \\
& Educativa & $(11.9 \%)$ & $(88.1 \%)$ \\
& Jurídica & 3 & 13 \\
Área de conocimiento & $(18.8 \%)$ & $81.2 \%$ \\
& Neuropsicología & 0 & 1 \\
& & 2 & $(100.0 \%)$ \\
& Organizacional & $(40.0 \%)$ & 3 \\
& Social-Comunitaria & 1 & $(60.0 \%)$ \\
& & $(25.0 \%)$ & $(75.0 \%)$ \\
& Psicometría & 2 & 9 \\
& & $(18.2 \%)$ & $(81.8 \%)$ \\
& Bibliometría & 4 & 12 \\
& & $(25.0 \%)$ & $(75.0 \%)$ \\
& & 0 & 2 \\
& & $(17.8 \%)$ & $(82.2 \%)$ \\
\hline
\end{tabular}

Tabla 6.

Uso de análisis correlaciónales de acuerdo con el área de conocimiento

\begin{tabular}{llcc}
\hline & & \multicolumn{2}{c}{ Uso de análisis correlacional } \\
\cline { 3 - 3 } & & No & Sí \\
& Adicciones & 3 & 1 \\
& Clínica-Salud & $(75.0 \%)$ & $(25.0 \%)$ \\
& & 22 & 20 \\
& Educativa & $(52.4 \%)$ & $(47.6 \%)$ \\
& Jurídica & 14 & 2 \\
& & $(87.5 \%)$ & $(12.5 \%)$ \\
& Neuropsicología & 1 & 0 \\
& & $(100.0 \%)$ & 1 \\
& Organizacional & 4 & $(20.0 \%)$ \\
& & $(80.0 \%)$ & 2 \\
& Social-Comunitaria & 2 & $(50.0 \%)$ \\
& & $(50.0 \%)$ & 3 \\
& Psicometría & 8 & $(27.3 \%)$ \\
& Bibliometría & $(72.7 \%)$ & 10 \\
& & 6 & $(62.5 \%)$ \\
& & $(37.5 \%)$ & 0 \\
\hline
\end{tabular}


En cuanto a los análisis multivariados, estos prevalecen seguido por los del área de psicometría (68.8\%) y organien los artículos bibliométricos y del área jurídica (100\%), zacional (50\%) (véase Tabla 8).

Tabla 7.

Uso de análisis inferencial de acuerdo con el área de conocimiento

\begin{tabular}{|c|c|c|c|}
\hline & & \multicolumn{2}{|c|}{ Uso de análisis inferencial } \\
\hline & & No & Sí \\
\hline & Adicciones & $\begin{array}{c}1 \\
(25.0 \%)\end{array}$ & $\begin{array}{c}3 \\
(75.0 \%)\end{array}$ \\
\hline & Clínica-Salud & $\begin{array}{c}9 \\
(21.4 \%)\end{array}$ & $33(78.6 \%)$ \\
\hline & Educativa & $\begin{array}{c}4 \\
(25.0 \%)\end{array}$ & $\begin{array}{c}12 \\
(75.0 \%)\end{array}$ \\
\hline & Jurídica & 0 & $\begin{array}{c}1 \\
(100.0 \%)\end{array}$ \\
\hline \multirow[t]{5}{*}{ Área de conocimiento } & Neuropsicología & $\begin{array}{c}1 \\
(20.0 \%)\end{array}$ & $\begin{array}{c}4 \\
(80.0 \%)\end{array}$ \\
\hline & Organizacional & $\begin{array}{c}2 \\
(50.0 \%)\end{array}$ & $\begin{array}{c}2 \\
(50.0 \%)\end{array}$ \\
\hline & Social-Comunitaria & $\begin{array}{c}4 \\
(36.4 \%)\end{array}$ & $\begin{array}{c}7 \\
(63.6 \%)\end{array}$ \\
\hline & Psicometría & $\begin{array}{c}5 \\
(31.2 \%)\end{array}$ & $\begin{array}{c}11 \\
(68.8 \%)\end{array}$ \\
\hline & Bibliometría & $\begin{array}{c}2 \\
(100.0 \%)\end{array}$ & 0 \\
\hline Total & & $\begin{array}{c}28 \\
(27.7 \%)\end{array}$ & $\begin{array}{c}73 \\
(72.3 \%)\end{array}$ \\
\hline
\end{tabular}

Tabla 8.

Uso de análisis multivariado de acuerdo con el área de conocimiento

\begin{tabular}{|c|c|c|c|}
\hline & & \multicolumn{2}{|c|}{ Uso de análisis multivariado } \\
\hline & & No & Sí \\
\hline & Adicciones & $\begin{array}{c}3 \\
(75.0 \%)\end{array}$ & $\begin{array}{c}1 \\
(25.0 \%)\end{array}$ \\
\hline & Clínica-Salud & $\begin{array}{c}28 \\
(66.7 \%)\end{array}$ & $\begin{array}{c}14 \\
(33.3 \%)\end{array}$ \\
\hline & Educativa & $\begin{array}{c}9 \\
(56.2 \%)\end{array}$ & $\begin{array}{c}7 \\
(43.8 \%)\end{array}$ \\
\hline & Jurídica & 0 & $\begin{array}{c}1 \\
(100.0 \%)\end{array}$ \\
\hline \multirow[t]{5}{*}{ Área de conocimiento } & Neuropsicología & $\begin{array}{c}4 \\
(80.0 \%)\end{array}$ & $\begin{array}{c}1 \\
(20.0 \%)\end{array}$ \\
\hline & Organizacional & $\begin{array}{c}2 \\
(50.0 \%)\end{array}$ & $\begin{array}{c}2 \\
(50.0 \%)\end{array}$ \\
\hline & Social-Comunitaria & $\begin{array}{c}7 \\
(63.6 \%)\end{array}$ & $\begin{array}{c}4 \\
(36.4 \%)\end{array}$ \\
\hline & Psicometría & $\begin{array}{c}5 \\
(31.2 \%)\end{array}$ & $\begin{array}{c}11 \\
(68.8 \%)\end{array}$ \\
\hline & Bibliometría & $\begin{array}{c}2 \\
(100.0 \%)\end{array}$ & 0 \\
\hline Total & & $\begin{array}{c}60 \\
(59.4 \%)\end{array}$ & $\begin{array}{c}41 \\
(40.6 \%)\end{array}$ \\
\hline
\end{tabular}


Las pruebas paramétricas son aplicadas en áreas como organizacional $(100 \%)$, neuropsicología $(80 \%)$, adicciones (75\%), psicometría y educativa $(68.8 \%)$, clínica-salud (64.3\%) y social-comunitaria $(63.6 \%)$, como las más relevantes; de la misma manera, las pruebas no paramétricas por su parte son aplicadas en clínica-salud (28.6\%), seguida por social-comunitaria (27.3\%) y organizacional y psicometría con $25 \%$ (Tabla 9).

Resultados de variables cuantitativas por área

En la Figura 20 se presentan los resultados del número de autores por área.

Tabla 9.

Uso de pruebas paramétricas y no paramétricas de acuerdo con el área de conocimiento

\begin{tabular}{|c|c|c|c|c|c|}
\hline & & \multicolumn{2}{|c|}{ Uso de pruebas paramétricas } & \multicolumn{2}{|c|}{ Uso de pruebas no paramétricas } \\
\hline & & No & Sí & No & Sí \\
\hline & Adicciones & $\begin{array}{c}1 \\
(25.0 \%)\end{array}$ & $\begin{array}{c}3 \\
(75.0 \%)\end{array}$ & $\begin{array}{c}4 \\
(100.0 \%)\end{array}$ & 0 \\
\hline & Clínica-Salud & $\begin{array}{c}15 \\
(35.7 \%)\end{array}$ & $\begin{array}{c}27 \\
(64.3 \%)\end{array}$ & $\begin{array}{c}30 \\
(71.4 \%)\end{array}$ & $\begin{array}{c}12 \\
(28.6 \%)\end{array}$ \\
\hline & Educativa & $\begin{array}{c}5 \\
(31.2 \%)\end{array}$ & $\begin{array}{c}11 \\
(68.8 \%)\end{array}$ & $\begin{array}{c}13 \\
(81.2 \%)\end{array}$ & $\begin{array}{c}3 \\
(18.8 \%)\end{array}$ \\
\hline & Jurídica & $\begin{array}{c}1 \\
(100.0 \%)\end{array}$ & 0 & $\begin{array}{c}1 \\
(100.0 \%)\end{array}$ & 0 \\
\hline \multirow[t]{5}{*}{ Área de conocimiento } & Neuropsicología & $\begin{array}{c}1 \\
(20.0 \%)\end{array}$ & $\begin{array}{c}4 \\
(80.0 \%)\end{array}$ & $\begin{array}{c}5 \\
(100.0 \%)\end{array}$ & 0 \\
\hline & Organizacional & 0 & $\begin{array}{c}4 \\
(100.0 \%)\end{array}$ & $\begin{array}{c}3 \\
(75.0 \%)\end{array}$ & $\begin{array}{c}1 \\
(25.0 \%)\end{array}$ \\
\hline & Social-Comunitaria & $\begin{array}{c}4 \\
(36.4 \%)\end{array}$ & $\begin{array}{c}7 \\
(63.6 \%)\end{array}$ & $\begin{array}{c}8 \\
(72.7 \%)\end{array}$ & $\begin{array}{c}3 \\
(27.3 \%)\end{array}$ \\
\hline & Psicometría & $\begin{array}{c}5 \\
(31.2 \%)\end{array}$ & $\begin{array}{c}11 \\
(68.8 \%)\end{array}$ & $\begin{array}{c}12 \\
(75.0 \%)\end{array}$ & $\begin{array}{c}4 \\
(75.0 \%)\end{array}$ \\
\hline & Bibliometría & $\begin{array}{c}2 \\
(100.0 \%) \\
\end{array}$ & 0 & $\begin{array}{c}2 \\
(100.0 \%) \\
\end{array}$ & 0 \\
\hline Total & & $\begin{array}{c}34 \\
(33.7 \%) \\
\end{array}$ & $\begin{array}{c}67 \\
(66.3 \%) \\
\end{array}$ & $\begin{array}{c}78 \\
(77.2 \%) \\
\end{array}$ & $\begin{array}{c}23 \\
(22.8 \%) \\
\end{array}$ \\
\hline
\end{tabular}



Número de autores

Figura 20. Número de autores por área 
Elárea en la que más autores se registran es neuropsicología con 3.8, y bibliometría con 3.5 autores en promedio, mientras que en jurídica hay 1 autor por artículo.

De acuerdo con los resultados, el año con mayor número de publicaciones fue 2014, con veintisiete artículos. Veinticinco es el promedio anual de artículos publicados durante los años analizados; para los próximos números se proyecta aumentar a treinta anuales.

Así mismo, ha predominado la publicación en idioma español; para los futuros números todos los artículos se publicarán también en inglés y portugués . Respecto al área de conocimiento, se observa que la psicología clínica y de la salud continúa siendo el campo con mayor número de artículos publicados. Dicho indicador ha llevado a que en algunas clasificaciones, como las que divulga el SCImago Journal y Country Rango, a partir de la información incluida en Scopus y cuyo indicador es el SCImago Journal Ranking (SJR), ubiquen a la Revista Acta en dos áreas de conocimiento: Psicología y Psiquiatría y Salud Mental.

En cuanto al tipo de investigación, se destaca que la mayoría son de tipo cuantitativo, y el tipo de muestreo que se registra es probabilístico, aunque el porcentaje más amplio no registra el método usado para la selección de la muestra. 2013 es el año en que la mayor parte de los artículos no evidencian el muestreo usado. En los años 2010, 2011 y 2012 (50\%, 45.8\% y 47.8\%, respectivamente) predomina el uso del muestreo probabilístico como herramienta de selección de la muestra; en 2014 sobresalen las investigaciones en las que se usó un muestreo no probabilístico (44.4\%).

Cabe destacar que en los análisis presentados no se tuvo en cuenta la procedencia geográfica e institución de los autores, los índices de colaboración entre instituciones, los énfasis temáticos de lo publicado, por lo cual se espera que para los próximos análisis se incluyan estos indicadores.

Otro aspecto que se espera cambiar en beneficio de los autores son los tiempos de demora entre la recepción y concepto de aceptación de publicación de los artículos postulados. Durante el periodo de observación se encontró que entre el tiempo de recibido el artículo y el concepto del mismo, hay un promedio de 190 días, mientras que el promedio entre el concepto y la aceptación es de 61 días. De igual manera, respecto a la procedencia de los artículos, se muestra que el $60.5 \%$ de los autores publicados durante los cinco años de observación corresponden a investigadores internacionales. En relación con esto, se mantendrá la tendencia de publicación de artículos internacionales con la finalidad de aumentar el posicionamiento geográfico y el fortalecimiento de las redes del concomimiento. Se espera también que en el futuro se incrementen los porcentajes de publicación institucional. En cuanto a la continuidad de estudios referentes a la caracterización de Acta, se fomentará la línea de investigación para la generación de estudios bibliométricos a partir de lo publicado y del comportamiento de las clasificaciones realizadas por diferentes sistemas de indexación, en los cuales se encuentre incluida Acta Colombiana de Psicología.

\section{REFERENCIAS}

Ávila-Toscano, J. H., Marenco-Escuderos, A., y Madariaga, C. (2014). Indicadores bibliométricos, redes de coautorías y colaboración institucional en revistas colombianas de psicología. Avances en Psicología Latinoamericana, 32(1), 167-182. Doi: dx.doi.org/10.12804/apl32.1.2014.12

Buela-Casal, G. y López-López, W. (2005). Evaluación de las Revistas Científicas Iberoamericanas de Psicología. Iniciativas y Estado Actual. Revista Latinoamericana de Psicología, 37 (1), 211-217.

Buela-Casal, G., Zych, I., Sierra, J.C. y Bermúdez, M.P. (2007). The Internationality Index of the Spanish Psychology Journals. International Journal of Clinical and Health Psychology, 7, 899-910.

Fava, G.A. y Montonary, A. (1997). Tendencias nacionales de investigación en psicología y psiquiatría (1981- 1995). Psicoterapia y Psicosomática, 66, 169- 174.

Fava, G. A, Ottolini, F. y Sonino, N. (2001). ¿Cuáles son los países líderes en investigación en medicina clínica? Una cita Análisis (desde 1981 hasta 1998). Psicoterapia y Psicosomática, 70, 283-287.

García Martínez, A. I., Guerrero Bote, V., Vargas Quesada, B. y De Moya-Anegón, F. (2008). La Psicología en el Cienciograma de los Países Iberoamericanos. Revista Latinoamericana de Psicología, 40 (3), 409-424

Jaraba-Barrios, B., Guerrero-Castro, J., Gómez-Morales, Y. y López-López, W. (2011). Bibliometría e historia de las prácticas académicas locales: un esbozo a partir del caso de la psicología en Colombia. Avances en Psicología Latinoamericana, 29(2), 354-369.

López-López, W. y Calvache, O. (1998). La psicología en habla hispana: 30 años de la revista latinoamericana de psicología. Revista Latinoamericana de Psicología, 30, 401-427.

López-López W., Silva, L.M., García-Cepero, M.C., AguilarBustamante, M.C. y Aguado-López, E. (2010). Panorama de la producción académica en la psicología colombiana. Acta Colombiana de Psicología, 13 (2), 35-46.

Patalano, M. (2005). Las publicaciones del campo científico: las revistas académicas de América Latina Anales de Documentación, 8, 217-235

Puche-Navarro, R. y Ossa, J. C. (2012). Claves de la Publicación Psicológica en Colombia: Ritmo, Grupos y Modalidades de Producción en la Práctica Investigativa. Revista Colombiana de Psicología, 21(1), 79-95. 
Rahman, M. y Fukui, T. (2000). Productividad de la investigación biomédica en los países asiáticos. Journal of Epidemioly, 10, 290-291.

Ravelo, E. (2007). Editorial: Logros alcanzados por Acta Colombiana de Psicología. Acta Colombiana de Psicología, $11(2), 5-6$.

Rivera-Garzón, DM (2008). Caracterización de la comunidad Científica de Psicología Que publica en la revista Universitas Psychologica (2002-2008). Universitas Psychologica, 7, 917932.

Russell, M., Ainsworth, S. y Narváez-Berthelemot, N. (2006). Colaboración científica de la Universidad Nacional Autónoma de México (UNAM) y su política institucional. Revista Española de Documentación Científica, 29(1), 56-73.
Sánchez López. M. y Vidal Vásquez. O. L. (2014). El impacto de divulgar el conocimiento científico como resultado de la investigación en la publicación de artículos. Revista Iberoamericana de Producción Académica y gestión Educativa, 1, 1-16

Vessuri, H. (1995). Estrategia de valoración de las revistas científicas latinoamericanas. In Cetto, A. M. y Hillerud, Kai-Inge (Comp.) Publicaciones cientificas en América Latina. Fondo de Cultura Económica. México. 200-210.

Zych I. y Buela-Casal G. (2007). Análisis comparativo de los valores en el Índice de Internacionalidad de las revistas iberoamericanas de psicología incluidas en la Web of Science. Revista Mexicana de Psicología, 24, 7-14.

Zych, I. y Buela-Casal, G. (2010). Internacionalidad de las revistas de psicología multidisciplinar editadas en Iberoamérica e incluidas en la Web of Science. Universitas Psychologica, 9 (1), 27-34. 